\section{International Scientific Journal Theoretical \& Applied Science}

\author{
p-ISSN: 2308-4944 (print) e-ISSN: 2409-0085 (online) \\ Year: $2015 \quad$ Issue: 02 Volume: 22 \\ Published: $28.02 .2015 \quad$ http://www.T-Science.org
}

SECTION 2. Applied mathematics. Mathematical modeling.
Razila Abzelhanovna Beshtaeva magistr of mathematics

Taraz State University named after M.H.Dulaty,

Kazakhstan razila-83@mail.ru

Nariman Malikovich Kiyalbekov teacher of mathematics

Specialized regional boarding-school for the especially gifted children of "Daryn" Kazakhstan nariman kiyalbekov@mail.ru

\title{
DISTRIBUTION OF HEAVY ADMIXTURE IN FRONTIER LAYER OF ATMOSPHERE AT NON-STATIONARY PENETRATING CONVECTION
}

Abstract: In work happens to semi theoretical and statistical theory to turbulent diffusion of the admixtures, is considered particularities of the process to diffusions in stratified on density to ambience. The Described methods of the experimental study to diffusions in ocean and atmosphere.

Key words: turbulent diffusion, coefficient of turbulent viscidity.

Language: Russian

Citation: Beshtaeva RA, Kiyalbekov NM (2015) DISTRIBUTION OF HEAVY ADMIXTURE IN FRONTIER LAYER OF ATMOSPHERE AT NON-STATIONARY PENETRATING CONVECTION. ISJ Theoretical \& Applied Science 02 (22): 29-35. doi: http://dx.doi.org/10.15863/TAS.2015.02.22.7

\section{РАСПРОСТРАНЕНИЕ ТЯЖЕЛОЙ ПРИМЕСИ В ПОГРАНИЧНОМ СЛОЕ АТМОСФЕРЫ ПРИ НЕСТАЦИОНАРНОЙ ПРОНИКАЮЩЕЙ КОНВЕКЦИИ}

Аннотация: В работе приводятся элементы полуэмпирических и статических теорий турбулентной диффузии примесей. Рассмотрены особенности процесса диффузии в стратифицированной по плотности среде. Описаны методы экспериментального исследования диффузии в океане и атмосфере.

Ключевые слова: турбулентная диффузия, коэффициент турбулентной вязкости.

В ряде прикладных задач особый интерес представляет изучение особенностей распространения пассивной примеси в условиях развитой атмосферной конвекции, когда из-за значительных скоростей подъема воздуха в термиках (струях и пузырях теплого воздуха) создаются условия для проникновения частиц примеси, в том числе и крупных, до уровня нижней кромки облаков. Эти частицы могут быть вовлечены в систему облачной конвекции, которая также характеризуется значительными вертикальными скоростями. По экспериментальным данным крупные частицы, скорость падения которых достигает десятков сантиметров в секунду, действительно имеются в облаках и, являясь ядрами коагуляции, влияют на процессы осадко-образования. Частицы, имеющие малую скорость падения (некоторые из них служат ядрами конденсации водяного пара), попав на большие высоты, могут разноситься ветром на расстояния порядка сотен и тысяч километров. Для описания процессов распространения примеси в пограничном слое атмосферы обычно используется полуэмпирическое уравнение турбулентной диффузии, в котором поля скорости ветра и коэффициента турбулентной диффузии рассчитываются из системы уравнений пограничного слоя. При этом нерегулярные мезомасштабные конвективные процессы в пограничном слое параметризуются как турбулентные (подсеточные). Однако решения, базирующиеся на таком подходе, не описывают многих важных особенностей структуры конвективного пограничного слоя.

Данная статья посвящена изучению некоторых особенностей распространения тяжелой пассивной примеси в условиях нерегулярной нестационарной мезомасштабной конвекции с использованием в качестве базовой гидродинамической модели конвективного ансамбля, а не параметрического представления данного процесса. Под тяжелой примесью будем понимать примесь, имеющую положительные скорости гравитационного оседания, не влияющую на движение основной среды, не 
вступающую в химические реакции. Источником поступления примеси является тонкий запыленный приземный слой атмосферы. Система уравнений, описывающая суточные вариации полей метеорологических элементов «сухого» конвективного ансамбля, развивающегося на фоне заданного горизонтально однородного крупномасштабного течения пограничного слоя Экмана, с учетом упрощений имеет вид

$$
\begin{gathered}
\frac{\partial \Omega}{\partial t}+(U+u) \frac{\partial \Omega}{\partial x}+w \frac{\partial \Omega}{\partial x}-w \frac{\partial^{2} U}{\partial z^{2}}=\frac{g}{\Theta_{0}} \frac{\partial \rho}{\partial x}+v \Delta \Omega, \\
\frac{\partial u}{\partial t}+(U+u) \frac{\partial V}{\partial x}+w \frac{\partial V}{\partial z}+w \frac{\partial V}{\partial z}=v \Delta V, \\
\frac{\partial V}{\partial t}+(U+u) \frac{\partial \rho}{\partial x}+w \frac{\partial \rho}{\partial x}+w \frac{\partial \Theta}{\partial z}=v \Delta \rho, \\
\Delta \Psi=\Omega, \quad w=\frac{\partial \Psi}{\partial x}, \quad U=-\frac{\partial \Psi}{\partial z}, \\
\Delta=\frac{\partial^{2}}{\partial x^{2}}+\frac{\partial^{2}}{\partial z^{2}}
\end{gathered}
$$

где $t$-время;

$x, y, z$ - горизонтальные и вертикальная координаты;

$u, v, w$ - конвективные компоненты скорости вдоль этих координат;

$U, V$ - экмановские компоненты скорости вдоль осей х и у;

$\Omega$ - вихрь;

$\Psi$ - функция тока; $\rho$ - отклонение потенциальной температуры от ее значения $\Theta$ в отсутствие конвекции;

$\Theta_{0}$ - среднее значение потенциальной температуры в конвективном слое;

$\boldsymbol{v}$ - коэффициент турбулентной вязкости, описывающий процессы подсеточного масштаба; $g$ - ускорение свободного падения.

Фоновое значения метеорологических элементов вычисляются из соотношений [5]

$$
\begin{aligned}
& U=U_{g}\left(1-\cos \xi e^{-\xi}\right), V=U_{g} \sin \xi e^{-\xi}, \quad \xi=\sqrt{\frac{\omega \sin \varphi}{v}} z, \\
& \Theta=\Theta_{0}+\gamma z+\Theta_{1} e^{-\eta} \sin (\omega t-\eta), \quad \eta=\sqrt{\frac{\omega}{2 v}} z
\end{aligned}
$$

где $U_{g}$ - скорость геострофического ветра;

$\gamma$ - градиент потенциальной температуры, соответствующий стандартной (устойчивой) атмосфере;

$\Theta_{1}$ - амплитуда суточного хода температуры подстилающей поверхности, которая предпологается известной функцией t;

$\omega$ - угловая скорость вращения Земли.

Система (1), (2) решатся численно при следующих граничных условиях:

$$
\psi=\Omega=\vartheta=0, z=0, H,
$$

где $z=H$ - верхняя граница, расположенная в устойчиво страцифицированной свободной атмосфере, куда конвекция не достигает. Заметим, что условие $\left.\Omega\right|_{z=0}=0 \quad$ не обеспечивает полностью выполнения условия прилипания и выбрано нами, как и в других работах $[5,8]$, исходя из удобства численной реализации задачи. Расчеты показали, что при выполнении условия $w=0$ интервал изменения и на нижней границе мал и не превышает $\pm 0,1 \mu / c$. В качестве условий на боковых границах $x=0, x=L$ используются условия периодичности. Предпологается, что в начальный момент $t=t_{0}$, соответствующий 9 ч местного времени, конвективные возмущения в плоскопараллельном потоке отсутствуют:

$$
\psi=\Omega=0 \text { при } t=t_{0},
$$

а конвекция возбуждается заданием в момент $t=t_{0}$ в каждом узле сетки на уровне $z=\Delta z_{1}$, некоторых значений $\rho$, выбираемых генератором случайных чисел из интервала 
[-1,1]. Другие подобные способы задания случайных пульсаций в $t=t_{0}$ приводят лишь к изменению времени адаптации (10-25 мин), после которого решение (в смысле средних характеристик) выходит на режим, зависящий лишь от параметров задачи $[5,8]$.

Для описания распространения примеси используем уравнение [1]

$$
\frac{\partial s}{\partial t}+(u+U) \frac{\partial s}{\partial x}+\left(w-w_{0}\right) \frac{\partial s}{\partial z}=\mu \Delta s,
$$

где $s$ - концентрация примеси;

$\mu$ - коэффициент турбулентной диффузии; $-\omega_{0}=$ const - скорость гравитационного оседания частиц примеси.

Будем считать, что примесь, поднимаемая микротурбулентными пульсациями ветра, поступает в атмосферу от загрязненной подстилающей поверхности. Для описания поступления частиц примеси с подстилающей поверхности используем граничное условие

$$
\mu \frac{\partial s}{\partial z}=-f \text { при } z=0,
$$

где $f>0$ - функция, параметризирующая процессы обмена примесью между землей и атмосферой. Хотя в общем случае функция $f$ зависит от многих факторов, в частности от приземной скорости ветра, состояния почвы, в данной работе для простоты примем $f=$ const . Будем также считать, что на больших высотах примесь отсутствует,

$$
s=0 \text { при } z=H,
$$

a на боковых поверхностях удовлетворяет условию периодичности. Заметим, что решение задачи (5)-(7) пропорционально $f$ из-за линейности исходных уравнений.

Следуя [14], покажем на примере аналитического решения (5)-(7), что условие (6) для $f=$ const обеспечивает преобладение более легких частиц по сравнению с более тяжелыми. Это решение в случае отсутствуия конвекции $(u=\omega=0)$ при нулевом начальном распределении примеси $(s=0$ при $t=0)$ имеет вид

$$
s=\frac{2 f}{\mu \sqrt{\pi}} \int_{\pi}^{\infty} \int_{\frac{\beta}{2 \sqrt{\mu t}}}^{\infty} \exp \left(-\alpha^{3}-\frac{\omega_{0} \beta}{2 \mu}\left(1+\frac{\omega_{0} \beta}{8 \mu \alpha^{2}}\right)\right) d \alpha d \beta
$$

Из (8) следует, что $s \rightarrow \infty$ при $\omega_{0} \rightarrow 0$ и $t \rightarrow \infty$.

Таким образом, условие (6) оказывается неприемлемым для изучения распространения очень легких частиц, поскольку концентрация их может неограниченно возрастать. Для тяжелых частиц полученное распределение с ростом $t$ достаточно быстро переходит в стационарное: $s=f / \omega_{0} \exp (-\omega z / \mu)$.

Очевидно, $s=f / \omega_{0}$ при $z=0, \quad$ и поэтому чем тяжелее частицы, тем меньше их в атмосфере.

Для задания начального распределения примеси при $u=\omega=0$ используется полученное выше стационарное аналитическое решение

$$
s=\frac{f}{\omega_{0}} \exp \left(-\frac{\omega_{0}}{\mu} z\right) \text { при } t=t_{0} .
$$

Учитывая отсутсвие надежных данных об $f$, расмотрим случай $f=$ const. Для удобства представления и интерпретации результатов расчетов воспользуемся нормировкой $s=s^{*} f / \omega_{0}$, при которой вид уравнения (5) не изменится, а условия (6), (9) соответственно перепишутся так: $\left(\mu / \omega_{0}\right) \partial s^{*} / \partial z=-1, s^{*}=\exp \left(-\omega_{0} z / \mu\right)$.

Поставленная задача решалась численно. Для интегрирования по времени была взята неявная схема Кранка - Николсона; производные по пространственным переменным расписывались с использованием «бокс» - метода. Получающаяся при этом система алгебраических уравнений решалась методом покомпонентного расщепления [6] с применением обычной прогонки по вертикали и циклической по горизонтали. Уравнение для функции тока $\psi$ решалось спектральноразностным методом с использованием быстрого преобразования Фурье. Решение отыскивалось в прямоугольной области с размерами 5120 м по $x \quad$ и $2448 \mu$ по $z(128 \times 48$ расчетных узлов) с постоянным шагом $\Delta x$ по горизонтали и переменными шагами по вертикали $\Delta z_{j}=a(j+b), j=1, \ldots, 48$.

В данной статье изложим результаты двух численных экспериментов для случаев нулевого 
фонового течения $\left(\mathrm{U}_{\mathrm{g}}=0\right)$ и слабого геострофического потока в атмосфере $\left(\mathrm{U}_{\mathrm{g}}=3 \mathrm{\mu} / \mathrm{c}\right)$. Основная цель расчетов - выявить различия в характере распространения примеси с различными скоростями падения частиц. Значения варьировались в диапазоне от 0,01 до 0,5 $\mathrm{M} / \mathrm{c}$. Остальные параметры брались следующими :

$$
\begin{gathered}
\Delta t=60 c ; \Delta x=40 \mu ; \quad \mathrm{a}=2 ; \mathrm{b}=1 ; \\
\gamma=4 \times 10^{-3}{ }^{0} \mathrm{C} / \mu ; \theta_{1}=4^{\circ} \mathrm{C} ; \theta_{0}=20^{\circ} \mathrm{C} ; \\
\mathrm{v}=\mu=5 \mathrm{~m}^{2} / c .
\end{gathered}
$$

Распространение примеси изучалось с 9 до 21 ч местного времени.

Вначале дадим краткую характеристику структуры конвективного ансамбля, полученого в результате численного моделирования. Элементами конвекции, переносящими примесь вверх, являются спонтанно возникающие в нижнем неустойчивом слое струи и пузыри теплого воздуха, причем число конвективных элементов в форме струй больше, чем в форме пузырей, что согласуется с данными [3]. Процесс спонтанного образования термиков происходит непрерывно с начала развития до затухания конвекции. Значение $\omega$ в струях достигает $4 \mathrm{~m} / \mathrm{c}$ между ними, где движения нисходящие, - $2 \mathrm{M} / \mathrm{c}$. Поэтому области восходящих движений узкие (150-200м), а нисходящих в среднем в 1,5-2 раза шире. Положительные значения отклонений температуры не превышают $0.6{ }^{\circ} \mathrm{C}$, отрицательные $\quad-0.3 \quad{ }^{\circ} \mathrm{C}$. Конвективный ансамбль, состоящий из взаимодействующих между собой струй и пузырей, обеспечивает хорошее перемешивание слоя, занятого конвекцией, делая его стратификацию почти безразличной. Ниже слоя перемешивания у самой земли где конвекция слабая, формируется слой толщиной 20-30м со сверхадиабатическими градиентами температуры. В верхней части слоя перемешивания образуется тонкий слой с большей, чем в сводной атмосфере, устойчивостью, так называемая «инверсионная шапка». Bce характеристики конвекции претерпевают временные изменения: с ростом температуры на земле $\left(\left.\theta\right|_{\mathrm{z}=0}\right)$ увеличиваются горизонтальные и вертикальные масштабы конвекции; отклонения температуры и скорости в элементах конвекции достигают максимума приблизительно в 15 , а затем с уменьшением $\left.\theta\right|_{\mathrm{z}=0}$ постепенно ослабевают. Влияние слабого ветра приводит к перемещению термиков вдоль оси $x$ со скоростью, приблизительно равной средней скорости ветра внутри конвективного слоя, а также к увеличению горизонтальных градиентов температуры и скорости на наветренной стороне термиков. Кроме того, заметно возрастают средние вертикальные и горизонтальные масштабы элементов конвекции.

Сопоставление как с данными наблюдений, так и с теоритическими работами $[5,8]$ показывает, что модель качественно правильно описывает особенности временной эволюции и структуры реальной проникающей конвекции.

Переходим теперь к изложению результатов численного моделирования распространения примеси в конвективных условиях. При расчетах выдавались значения $s^{*}$, осредненные по $x$ и $t$ вертикальные профили безразменной концентрации $\bar{s}$, суммарный безразмерный вертикальный поток примеси $F$ и его составляющие вследствие турбулентной диффузии $F_{1}$, конвективного переноса $F_{2}$, гравитационного оседания $F_{3}$, а также величина $<s>$, пропорциональная общей массе примеси, содержащейся в области решения:

$$
\begin{gathered}
\bar{s}=\frac{1}{T_{0} L} \int_{t}^{t+T_{0}} \int_{0}^{L} s^{*} d x d t^{\prime}, F=F_{1}+F_{2}+F_{3}, F_{1}=\frac{v}{\omega_{0}} \frac{\partial \bar{s}}{\partial z}, \\
F_{2}=\frac{1}{T_{0} L \omega_{0}} \int_{t}^{t+T_{0}} \int_{0}^{L} s^{*} d x d t^{\prime}, F_{3}=-\bar{s},\left\langle\bar{s}>=\int_{0}^{H} \bar{s} d z .\right.
\end{gathered}
$$

Интервал осреднения по времени тот же, что и в $[10], T_{\mathrm{o}}=20$ мин. величины $\bar{s}, F$ и $\langle\bar{s}\rangle$ удовлетворяют соотношениям

$$
\frac{\partial \bar{s}}{\partial t}+\omega_{0} \frac{\partial F}{\partial z}=0
$$

$$
\frac{d<\bar{s}>}{\partial t}=\omega_{0}\left(1-\left.\bar{s}\right|_{z=0}\right),
$$

полученным осреднением (5) по формулам (6), (7), (10) с учетом соотношения $s^{*}=s \omega_{0} / f$. На рис.1a нанесены значения $S$ для частиц с различной скоростью падения. Пунктирные 
линии соответствует $\mathrm{t}=9 \mathrm{q}$, когда конвекция отсутствует и реализуется только диффузионно - гравитационный механизм переноса примеси 6 сплошные линии - стадии максимального развития конвекции ( $\mathrm{t}=15$ ч). Из рисунка следует, что при конвекции конценттрация примеси убывает с высотой в неустойчивом слое и почти постоянна в перемешанном. Сравнение решения (9) с численным, соответствующим $\mathrm{t}=15$ ч, позволяет сделать следующий вывод: чем тяжелее частицы примеси, тем больших высот они достигают в условиях конвекции по сравнению со случаем без конвекции. Так, частицы с $\omega_{0}=0,5 ; 0,1 ; 0,02 \mu / c$ достигают высот порядка $1 \kappa м$, в то время как без конвекции они достигают высот 50, 200 и 900 м соответственно. Отношение общей массы примеси в атмосфере при $\mathrm{t}=154$ к своему значению при $\mathrm{t}=9$ ч для $\omega_{0}=0,5,0,1,0,02$

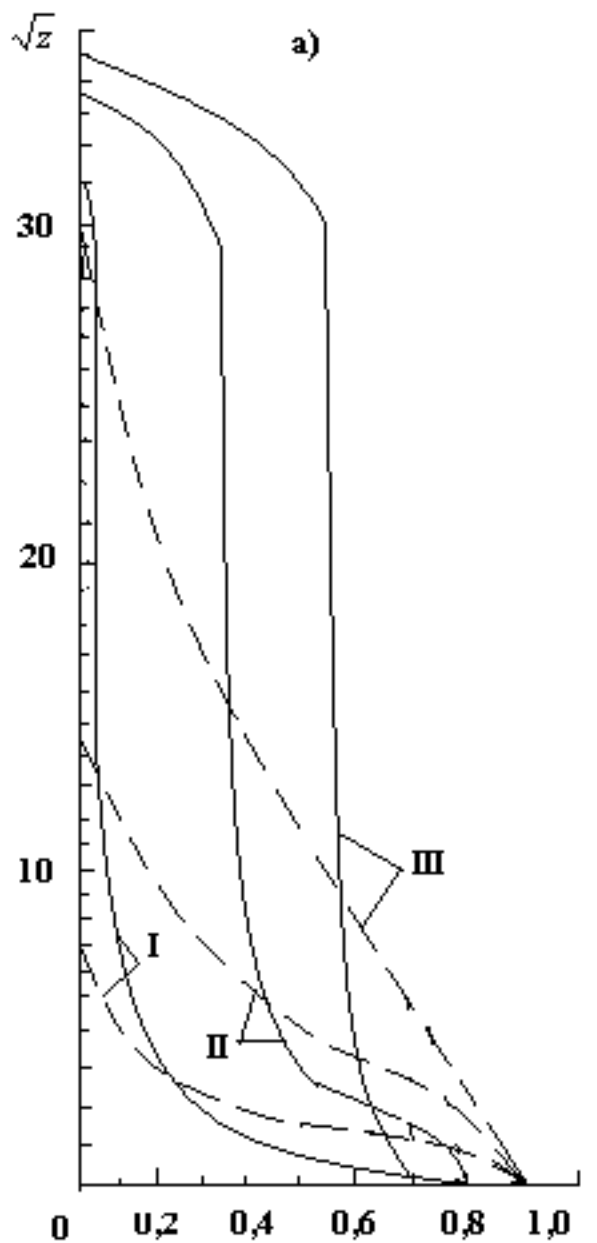

м/с составляет 5,$7 ; 4,1 ; 1,2$ соответственно. Таким образом, особенно ощутимо загрязнение воздуха частицами крупных размеров, которые влияют на радиационные притоки тепла и прозрачность атмосферы. Отметим, что одним из основных признаков условий развитой конвекции при слабом внешнем ветре либо отсутствии его (идеальной летной погоды для планеристов) является замутненность нижнего слоя атмосферы толщиной около 1 kм и более [4]. Что касается концентрации примеси $\bar{S}$ на небольших высотах, то в условиях без конвекции она оказывается более значительной, т. е. несмотря на увеличение общей массы примеси в воздухе, конвекция очищает слой атмосферы за счет загрязнения верхних. Это особенно хорошо заметно для частиц с $w \leq 0,1 \mu / c$.

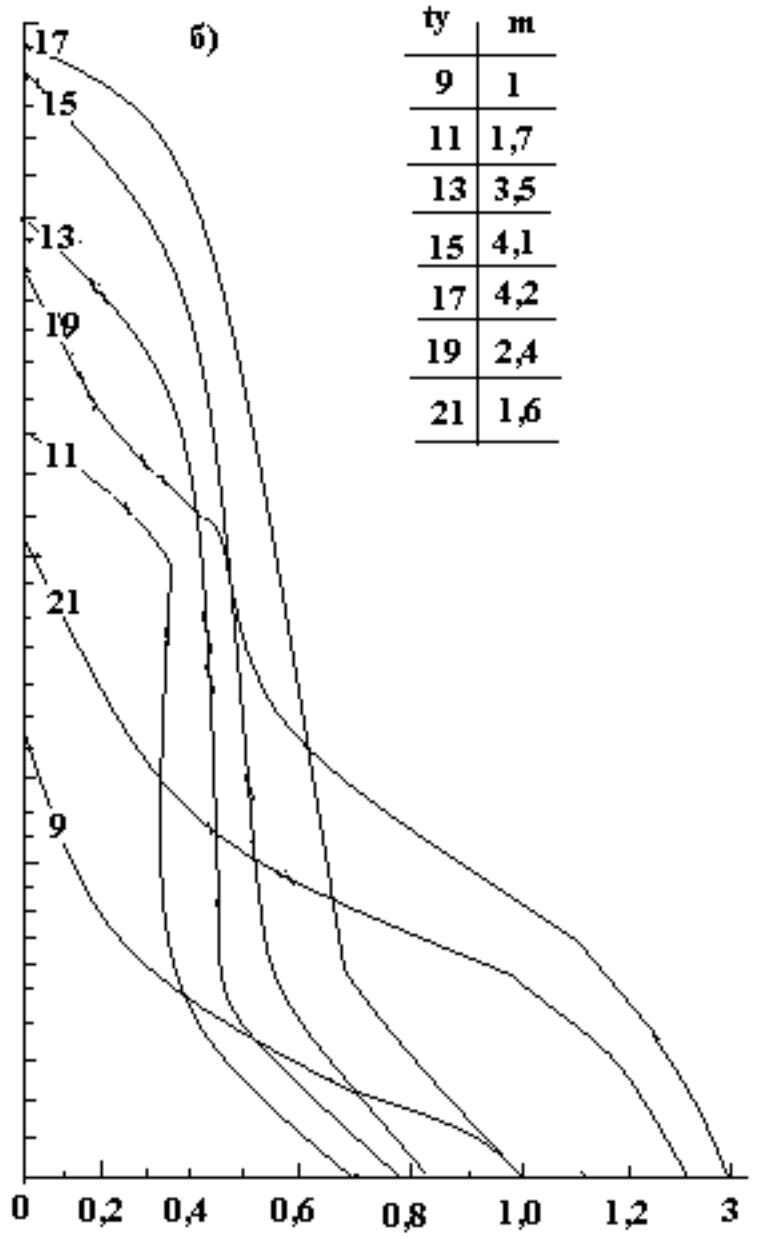

Рисунок 1 - Профили осредненной по $x_{\text {и }} t$ безразмерной концентрации $\bar{s}$ для $U_{g}=0$ :

a) $\omega_{0}=0,02$ (III). 0,1 (II), 0,5 $\mathcal{M} / c$ (I). б) $\omega_{0}=0,1 \quad \mu / c$ : цифры на кривых - время в часах; $m$ отношение общей массы примеси к своему значению в $t=9 u$. 
Из рис.1б, на котором для различных моментов времени нанесены профили $\bar{s}$ при $\omega_{0}=0,1 \mu / c$, видно, что по мере развития конвекции концентрация примеси у земли сначала убывает до 11 , потом увеличивается до 19 ч, а затем снова уменьшается, стремясь к своему значению при $\mathrm{t}=9$ ч. В перемешанном слое концентрации $\bar{s}$ вначале растет до $t \approx 17 u$, а потом убывает. Общая масса примеси, как это следует из (12), также увеличивается до 174 , пока $\left.\bar{s}\right|_{z=0}<1$, а затем, когда $\left.\bar{S}\right|_{z=0}>1$, начинает убывать.

Изменение во времени величины $m$, равное отношению общей массы примеси к своему значению в $t=9$, для $\omega_{0}=0,1 \mathrm{M} / \mathrm{c}$ показано в табл. На рис. 1б. Отметим также, что в стадии развития (значения $\langle\bar{s}\rangle$ растет) в верхней части перемешанного слоя концентрация быстро затухает с высотой и в инверсионный слой тяжелая примесь почти не проникает. Похожую картину дают данные наблюдений [7], согласно которым между слоем перемешивания внизу и чистым воздухом вверху часто имеется резкая граница, расположенная под инверсионным слоем. Стадия затухания (значение $\langle\bar{s}\rangle$ убывает) начинается с размывания этой границы. Кроме того, на стадии развития решение может иметь локальный максимум в верхней части слоя перемешивания. На рис. 16 слабые локальные максимумы видны для $t=11$, 13 ч. Для мгновенных значений примеси, не осредненных по времени, локальный максимум может достигать $30 \%$ от значения в слое перемешивания.

На высотах, не превышающих 20 м, превалирующей во время конвекции является турбулентная диффузия $\mathrm{F}_{1}$ (без конвекции $\mathrm{F}_{1}=-\mathrm{F}_{3}$ ). Выше поток $F_{1}$ затухает, а примесь переносится потоками $\mathrm{F}_{2}$ и $\mathrm{F}_{3}$. Причем на стадии развития конвективный поток $\mathrm{F}_{2}$ является превалирующим: $\quad F \approx F_{2}+F_{3}<0$. Отрицательные значения F появляются в 17 ч в верхней части конвективного слоя, т. е. по мере ослабления конвективных движений слой перемешивания примеси начинает разрушаться со своей верхней части, что хорошо видно на рис. 16, к 19 ч $\mathrm{F}<0$ уже во всем слое.

Показанные на рис. 1 результаты относятся к осредненным характеристикам распространения примеси. Вместе с тем интересно знать детальную структуру поля примеси при конвекции. Видно, что внутри термиков $(\omega>0)$ концентрация примеси в основном выше, чем вне их. Наличие такой корреляции при конвекции, по видимому, дает возможность планеристам прослеживать визуально наиболее мощные термики [4].

При включении в модель слабого геострофического ветра $\left(U_{g}=3 \mathrm{M} / \mathrm{c}\right)$ результаты мало чем отличались от изложенных выше. Происходило небольшое увеличение значений в перемешанном слое из-за незначительной интенсификации конвекции.

Подводя итог вышесказанному, отметим, что на базе данной модели рассмотрено распространение тяжелой пассивной примеси в заданной метеорологической ситуации, благоприятной для развития проникающей конвекции. Получено, с одной стороны, качественное объяснение известных экспериментальных фактов: замутненность нижнего слоя атмосферы толщиной около $1 \mathrm{~km}$ при конвекции; большая концентрация примеси внутри термиков, чем вне их; проникновение крупных частиц примеси до нижней кромки облаков. С другой стороны, выявлены новые интересные характеристики процесса переноса примеси от запыленного приземного слоя: наличие слоя перемешивания в вертикальном профиле концентрации примеси, временной ход концентрации у земли в зависимости от интенсивности конвекции. Кроме того, показана степень влияния динамического и диффузионного факторов на перенос частиц примеси в зависимости от времени и высоты. Поскольку получение данных о распространении примеси в реальной атмосфере связано с известными материальными и техническими трудностями, то предложенный подход может оказаться полезным для разработки процедур параметризации процесса переноса примеси.

References: 
1. Leonenko OI, Zil'bershteyn OI (1991) Modelirovanie rasprostraneniya neftyanykh uglevodorodov v Burgasskom zalive Chernogo morya. - Trudy GOINA, vyp.197, 1991, pp. 149-155.

2. Marchuk GI (1997) Chislennye metody prognoze pogody. -L.: Gidrometeoizdat, 1997. $-360 \mathrm{p}$.

3. Marchuk GI (1994) Chislennoe reshenie zadach dinamiki atmosfery $i$ okeana. $-\mathrm{L}$.: Gidrometeoizdat, 1994. -303 p.

4. Marchuk GI, Kagan BA (1983) Dinamika okeanicheskikh prilivov. -L.: Gidrometeoizdat, 1983. -360p.

5. Sarkisyan AS (1987) Chislennyy analiz i prognoz morskikh techeniy. $-\mathrm{L}$.: Gidrometeoizdat, 1987. -184p.
6. Samarskiy AA (1989) Teoriya raznostnykh skhem. - Moscow: Nauka,1989. -552p.

7. Samarskiy AA (1997) Matematicheskoe modelirovanie. - Moscow: Nauka,1997. -303p.

8. Kurant R, Gil'bert D (1951) Metody matematicheskoy fiziki. T.1,2 -Moscow: L.: GITTL.

9. Zenkeevich O (1985) Metod konechnykh elementov v tekhnike. -Moscow: Mir, 1985. $544 \mathrm{p}$.

10. Mezinger F, Arakova A (1979) Chislennye metody ispol'zuemye $\mathrm{V}$ atmosfernykh modelyakh. -L.: Gidrometeoizdat, 1979. -136p. 